\title{
MULTI-OBJECTIVE OPTIMIZATION PROBLEM WITH BOUNDED PARAMETERS
}

\author{
Ajay Kumar BhurJeE ${ }^{1}$ and Geetanjali Panda ${ }^{1}$
}

\begin{abstract}
In this paper, we propose a nonlinear multi-objective optimization problem whose parameters in the objective functions and constraints vary in between some lower and upper bounds. Existence of the efficient solution of this model is studied and gradient based as well as gradient free optimality conditions are derived. The theoretical developments are illustrated through numerical examples.
\end{abstract}

Keywords. Multi-objective optimization problem, efficient solution, optimality condition, interval valued convex function.

Mathematics Subject Classification. 90C25, 90C29, 90C30.

\section{INTRODUCTION}

In most of the real-life optimization models, the parameters in the objective function and constraints are not known exactly due to the presence of improper information in the data set. The lower and upper bounds of the parameters can be estimated from the historical data. In other words, the parameters are not fixed and assumed to lie in closed intervals. In that case, the objective and constraint functions map from real space to the set of intervals. So these functions are interval valued functions. In this paper, we address these type optimization models with several conflicting objective functions and call these optimization models as multiobjective interval optimization problem, in short $(M I O P)$. Such type situation appears in production planning, portfolio selection, transportation models etc. Example 1 explains such a model related to portfolio optimization.

Received September 5, 2013. Accepted April 11, 2014.

1 Department of Mathematics, Indian Institute of Technology Kharagpur, 721302, India. geetanjali@maths.iitkgp.ernet.in 
Example 1. Consider a portfolio management problem which has $n$ number of risky assets. Due to uncertainty in the market, the returns of the assets cannot be predicted exactly. From the historical data one can estimate the upper and lower bound of the parameters of the return in a fixed time period. Hence the expected return can be described in terms of interval parameters for a fixed time period. Let $x_{j}$ be the proportion of the total fund invested on $j$ th asset. Return of $j$ th asset lies between $r_{j}^{L}$ and $r_{j}^{R},\left(r_{j}^{L}<r_{j}^{R}\right)$, which is found from previous data. Since the return of all assets are lying in intervals, so the standard deviation and correlation between any two of them will also lie in intervals. Suppose $\mathbf{Q}_{M}=\left(\mathbf{Q}_{i j}\right)_{n \times n}$ denotes the $n \times n$ symmetric covariance interval matrix $\left(\mathbf{Q}_{i j}=\left[q_{i j}^{L}, q_{i j}^{R}\right]\right)$ corresponding to $i$ th and $j$ th asset. In this circumstance, the expected return and the variance of the resulting portfolio $x=\left(x_{1}, x_{2}, \ldots, x_{n}\right)^{T}$, are $\sum_{i}\left[r_{i}^{L}, r_{i}^{R}\right] x_{i}$ and $\sum_{i, j}\left[q_{i j}^{L}, q_{i j}^{R}\right] x_{i} x_{j}$, respectively.

In order to maximize the expected return and minimize the risk factor of the portfolio simultaneously, it is necessary to solve the interval multi-objective optimization problem,

$$
\begin{aligned}
& \min \left(-\sum_{i}\left[r_{i}^{L}, r_{i}^{R}\right] x_{i},\left(\sum_{i, j}\left[q_{i j}^{L}, q_{i j}^{R}\right] x_{i} x_{j}\right)^{\frac{1}{2}}\right) \\
& \text { subject to } \sum_{i} x_{i}=1, x_{i} \geq 0, i=1,2, \ldots, n .
\end{aligned}
$$

This is a nonlinear multi-objective interval optimization model.

Multi-objective optimization problems with interval parameters are studied by many researchers during last two decades (see $[1,3,4,8,9,11]$ ). The optimization models of all these papers have linear interval valued functions. Some recent developments in the area of nonlinear multi-objective interval optimization problem(MIOP) are due to Dunwei Gong et al. [2], Soares et al. [10] and Wu [13]. Dunwei Gong et al. [2] have suggested an interactive evolutionary algorithm for $(M I O P)$. This algorithm periodically provides a set of non-dominated solutions. GL Soares et al. [10] have considered a robust multi-objective optimization problem with interval valued function as an inclusion of real valued function. In both the papers conditions for the existence of solution of $(M I O P)$ has not been studied. $\mathrm{Wu}[13]$ has studied the conditions for the existence of solution of a (MIOP) whose objective functions are interval valued functions and all constraints are real valued functions. This paper discusses the conditions for the existence of solution of $(M I O P)$ model whose objective functions as well as constraints are nonlinear interval valued functions. For this purpose approach of this paper is different from above approaches. The existence results use the concept of convexity and differentiability of a general interval valued function and derives the sufficient optimality

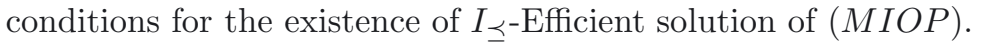

Development of the paper is explained in several sections. Section 2 discusses some prerequisites on interval analysis. In Section 3, sufficient optimality condition 
for $(M I O P)$ and the existence of solution is studied. The theoretical developments are illustrated in several numerical examples.

The following notations are used throughout the paper.

Bold capital letters denote closed intervals; $I(R)=$ The set of all closed intervals in $R ;(I(R))^{k}=$ The product space $\underbrace{I(R) \times I(R) \times \ldots \times I(R)} . \mathbf{C}_{v}^{k}=$ $k$ times

$k$-dimensional column vector, whose elements are intervals; $\mathbf{C}_{v}^{k} \in(I(R))^{k}, \mathbf{C}_{v}^{k}=$ $\left(\mathbf{C}_{1}, \mathbf{C}_{2}, \ldots, \mathbf{C}_{k}\right)^{T}, \mathbf{C}_{j}=\left[c_{j}^{L}, c_{j}^{R}\right] ; c_{j}^{L}, c_{j}^{R} \in R, j \in \Lambda_{k}, \Lambda_{k}=\{1,2, \ldots, k\} ;$ The degenerate interval $\hat{\eta}$ is denoted by $\hat{\eta}=[\eta, \eta]$, where $\eta$ is a real number.

\section{Preliminaries}

For two real vectors $a=\left(a_{1}, a_{2}, \ldots, a_{n}\right)^{T}, b=\left(b_{1}, b_{2}, \ldots, b_{n}\right)^{T}$ in $R^{n}$, we denote $a \geqq_{v} b \Leftrightarrow a_{i} \geq b_{i} ; a \leqq_{v} b \Leftrightarrow a_{i} \leq b_{i} ; a>_{v} b \Leftrightarrow a_{i}>b_{i} ; a<_{v} b \Leftrightarrow a_{i}<b_{i}, i \in \Lambda_{n}$.

Let $* \in\{+,-, \cdot, /\}$ be a binary operation on the set of real numbers. The binary operation $\circledast$ between two intervals $\mathbf{A}=\left[a^{L}, a^{R}\right]$ and $\mathbf{B}=\left[b^{L}, b^{R}\right]$ in $I(R)$, denoted by $\mathbf{A} \circledast \mathbf{B}$ is the set $\{a * b: a \in \mathbf{A}, b \in \mathbf{B}\}$. In case of division $(\mathbf{A} \oslash \mathbf{B})$, it is assumed that $0 \notin \mathbf{B}$. These interval operations can also be expressed in terms of parameters. Any point in $\mathbf{A}$ may be expressed as $a(t)=a^{L}+t\left(a^{R}-a^{L}\right), t \in[0,1]$. An interval $\mathbf{A}$ is said to be positive interval if $a(t)$ is positive for every $t$. Algebraic operations of intervals may be explained in parametric form as follows.

$$
\mathbf{A} \circledast \mathbf{B}=\left\{a\left(t_{1}\right) * b\left(t_{2}\right) \mid t_{1}, t_{2} \in[0,1]\right\}
$$

An interval vector $\mathbf{C}_{v}^{k} \in(I(R))^{k}$ may be expressed in terms of parameters as

$$
\begin{aligned}
\mathbf{C}_{v}^{k} & =\left\{c(t) \mid c(t)=\left(c_{1}\left(t_{1}\right), c_{2}\left(t_{2}\right), \ldots, c_{k}\left(t_{k}\right)\right)^{T},\right. \\
t & =\left(t_{1}, t_{2}, \ldots, t_{k}\right)^{T}, c_{j}\left(t_{j}\right) \in \mathbf{C}_{j}, \\
c_{j}\left(t_{j}\right) & \left.=c_{j}^{L}+t_{j}\left(c_{j}^{R}-c_{j}^{L}\right), t_{j} \in[0,1], j \in \Lambda_{k}\right\}
\end{aligned}
$$

The set of intervals $I(R)$ is not a totally order set. Partial ordering between two intervals can be represented in parametric form. For $\mathbf{A}, \mathbf{B} \in I(R)$,

$\mathbf{A} \preceq \mathbf{B}$ if $a(t) \leq b(t), \forall t \in[0,1]$ and $\mathbf{A} \prec \mathbf{B}$ if $a(t)<b(t), \quad \forall t \in[0,1]$

$$
\mathbf{A}=\mathbf{B} \text { if } a(t)=b(t), \quad \forall t \in[0,1]
$$

Note that $\mathbf{A} \preceq \mathbf{B}$ is not same as $\mathbf{B} \ominus \mathbf{A} \succeq \mathbf{0}$. For example $[2,5] \preceq[3,7]$, but $[3,7] \ominus[2,5]=[-2,5] \nsucceq \mathbf{0}$.

Next we summarize interval valued function and some of its properties below which are due to [1]. 
For $c(t) \in \mathbf{C}_{v}^{k}$, let $f_{c(t)}: R^{n} \rightarrow R$. For a given interval vector $\mathbf{C}_{v}^{k}$, an interval valued function $\mathbf{F}_{\mathbf{C}_{v}^{k}}: R^{n} \rightarrow I(R)$ can be expressed in the parametric form as

$$
\mathbf{F}_{\mathbf{C}_{v}^{k}}(x)=\left\{f_{c(t)}(x) \mid f_{c(t)}: R^{n} \rightarrow R, c(t) \in \mathbf{C}_{v}^{k}\right\}
$$

For every fixed $x$, if $f_{c(t)}(x)$ is continuous in $t$ then $\min _{t \in[0,1]^{k}} f_{c(t)}(x)$ and $\max _{t \in[0,1]^{k}} f_{c(t)}(x)$ exist. In that case

$$
\mathbf{F}_{\mathbf{C}_{v}^{k}}(x)=\left[\min _{t \in[0,1]^{k}} f_{c(t)}(x), \max _{t \in[0,1]^{k}} f_{c(t)}(x)\right] .
$$

If $f_{c(t)}(x)$ is linear in $t$ then $\min _{t \in[0,1]^{k}} f_{c(t)}(x)$ and $\max _{t \in[0,1]^{k}} f_{c(t)}(x)$ exist in the set of vertices of $\mathbf{C}_{v}^{k}$. If $f_{c(t)}(x)$ is monotonically increasing in $t$, then $\mathbf{F}_{\mathbf{C}_{v}^{k}}(x)=$ $\left[f_{c(0)}(x), f_{c(1)}(x)\right]$. The partial derivatives of $\mathbf{F}_{\mathbf{C}_{v}^{k}}: R^{n} \rightarrow I(R)$ at $x^{*}$ is calculated as follows.

$$
\frac{\partial \mathbf{F}_{\mathbf{C}_{v}^{k}}\left(x^{*}\right)}{\partial x_{i}}=\left\{\frac{\partial f_{c(t)}\left(x^{*}\right)}{\partial x_{i}} \mid \text { for every } t \in[0,1]^{k}, c(t) \in \mathbf{C}_{v}^{k}\right\} .
$$

If $\frac{\partial f_{c(t)}\left(x^{*}\right)}{\partial x_{i}}$ is continuous in $t$ then

$$
\frac{\partial \mathbf{F}_{\mathbf{C}_{v}^{k}}\left(x^{*}\right)}{\partial x_{i}}=\left[\min _{t \in[0,1]^{k}} \frac{\partial f_{c(t)}\left(x^{*}\right)}{\partial x_{i}}, \max _{t \in[0,1]^{k}} \frac{\partial f_{c(t)}\left(x^{*}\right)}{\partial x_{i}}\right] .
$$

The gradient of an interval valued function, $\mathbf{F}_{\mathbf{C}_{v}^{k}}: R^{n} \rightarrow I(R)$ at $x=x^{*}$ is an interval vector,

$$
\nabla \mathbf{F}_{\mathbf{C}_{v}^{k}}\left(x^{*}\right)=\left(\frac{\partial \mathbf{F}_{\mathbf{C}_{v}^{k}}\left(x^{*}\right)}{\partial x_{1}}, \frac{\partial \mathbf{F}_{\mathbf{C}_{v}^{k}}\left(x^{*}\right)}{\partial x_{2}}, \ldots, \frac{\partial \mathbf{F}_{\mathbf{C}_{v}^{k}}\left(x^{*}\right)}{\partial x_{n}}\right)^{T} .
$$

Using the representation of the partial ordering and interval valued function we can define interval valued convex function as follows.

Definition 2.1. Interval valued convex function.

Suppose $D \subseteq R^{n}$ is a convex set. For given $\mathbf{C}_{v}^{k} \in(I(R))^{k}$, the interval valued function $\mathbf{F}_{\mathbf{C}_{v}^{k}}: D \rightarrow I(R)$ is said to be convex with respect to $\preceq$ if for every $x_{1}, x_{2} \in D$ and $0 \leq \lambda \leq 1$,

$$
\mathbf{F}_{\mathbf{C}_{v}^{k}}\left(\lambda x_{1}+(1-\lambda) x_{2}\right) \preceq \lambda \mathbf{F}_{\mathbf{C}_{v}^{k}}\left(x_{1}\right) \oplus(1-\lambda) \mathbf{F}_{\mathbf{C}_{v}^{k}}\left(x_{2}\right) .
$$

Remark 2.2. From (2.2) and definition of interval valued convex function, one may observe that $\mathbf{F}_{\mathbf{C}_{v}^{k}}$ is convex with respect to $\preceq$ means $f_{c(t)}\left(\lambda x_{1}+(1-\lambda) x_{2}\right) \leq$ $\lambda f_{c(t)}\left(x_{1}\right)+(1-\lambda) f_{c(t)}\left(x_{2}\right)$, for all $t \in[0,1]^{k}$. So we can conclude that $\mathbf{F}_{\mathbf{C}_{v}^{k}}$ is convex with respect to $\preceq$ if and only if $f_{c(t)}(x)$ is a convex function on $D$ for every $t \in[0,1]^{k}$. 
The following separation theorem is needed to prove the existence of the solution of $(M I O P)$.

Proposition 2.3 [6]. Let $f$ be a m-dimensional convex vector function on the convex set $\Gamma \subset R^{n}$. Then either

(I) $f(x)<_{v} 0$ has a solution $x \in \Gamma$ or $(\mathbf{I I}) p^{T} f(x) \geq 0$ for all $x \in \Gamma$ for some $p \geqq v 0, p \in R^{m}$ but never both.

\section{Methodology}

We propose a general multi-objective interval optimization problem as,

$$
\begin{array}{ll}
(M I O P) \quad \min & \mathbf{F}(x) \\
\text { subject to } & \mathbf{G}_{D_{v}^{p} m_{p}}^{p}(x) \preceq(\text { or } \succeq) \mathbf{B}_{p}, p \in \Lambda_{q},
\end{array}
$$

where $\mathbf{F}(x)=\left(\mathbf{F}_{\mathbf{C}_{v}^{k_{1}}}^{1}(x), \mathbf{F}_{\mathbf{C}_{v}^{k_{2}}}^{2}(x), \ldots, \mathbf{F}_{\mathbf{C}_{v}^{k_{m}}}^{m}(x)\right)^{T}, \mathbf{F}_{\mathbf{C}_{v}^{k_{i}}}^{i}, \mathbf{G}_{D_{v}^{m_{p}}}^{p}: R^{n} \rightarrow I(R)$, $i \in \Lambda_{m}$, partial orderings in the constraints (3.5) are as defined in (2.2).

Using expression (2.4), the interval valued functions $\mathbf{F}_{\mathbf{C}_{v}^{k_{i}}}^{i}$ can be represented in the parametric form as

$$
\mathbf{F}_{\mathbf{C}_{v}^{k_{i}}}^{i}(x)=\left\{f_{c_{i}\left(t_{i}\right)}^{i}(x) \mid f_{c_{i}\left(t_{i}\right)}^{i}: R^{n} \rightarrow R, c_{i}\left(t_{i}\right) \in \mathbf{C}_{v}^{k_{i}}\right\} .
$$

Using Expression (2.4) and Inequality (2.2) the constraints of (MIOP) can be expressed as

$$
\left\{x \in R^{n} \mid \mathbf{G}_{D_{v}^{m_{p}}}^{p}(x) \preceq \mathbf{B}_{p}\right\}=\left\{x \in R^{n} \mid g_{d_{p}\left(t_{p}^{\prime}\right)}^{p}(x) \leq b\left(t_{p}^{\prime}\right)\right\},
$$

where $g_{d_{p}\left(t_{p}^{\prime}\right)}^{p}: R^{n} \rightarrow R, d_{p}\left(t_{p}^{\prime}\right) \in \mathbf{D}_{v}^{m_{p}}, b\left(t_{p}^{\prime}\right) \in \mathbf{B}_{p}$

Throughout this section, we consider $t=\left(t_{1}, t_{2}, \ldots, t_{m}\right)^{T}, t_{i}=\left(t_{i}^{1}, t_{i}^{2} \ldots, t_{i}^{k_{i}}\right)^{T}$, $t_{i}^{j} \in[0,1], j \in \Lambda_{k_{i}}, i \in \Lambda_{m}, t_{p}^{\prime} \in[0,1]$.

The feasible set for $(M I O P)$ can be expressed as the set,

$$
\begin{aligned}
S & =\left\{x \in R^{n} \mid \mathbf{G}_{D_{v}^{m_{p}}}^{p}(x) \preceq(\text { or } \succeq) \mathbf{B}_{p}, p \in \Lambda_{q}\right\} \\
& =\bigcap_{p \in \Lambda_{q}}\left\{x \in R^{n} \mid g_{d_{p}\left(t_{p}^{\prime}\right)}^{p}(x) \leq(\text { or } \geq) b_{p}\left(t_{p}^{\prime}\right), t_{p}^{\prime} \in[0,1]\right\} .
\end{aligned}
$$

Using expression (2.4), (MIOP) can be rewritten as

$$
\min _{x \in S}\left\{\left(f_{c_{1}\left(t_{1}\right)}^{1}(x), f_{c_{2}\left(t_{2}\right)}^{2}(x), \ldots, f_{c_{m}\left(t_{m}\right)}^{m}(x)\right) \mid c_{i}\left(t_{i}\right) \in \mathbf{C}_{v}^{k_{i}}, i \in \Lambda_{m}\right\} .
$$

Since $(M I O P)$ has several interval valued conflicting objective functions, so exact solution of $(M I O P)$ may not exist which minimizes all objective functions 
simultaneously. Like general multi-objective problem, solution of $(M I O P)$ is a compromise/ Pareto optimal/ efficient solution. For every $x$, the objective value of $(M I O P)$ is an interval vector. So, for any two different feasible points $x$ and $y$, the objective values $\mathbf{F}(x)$ and $\mathbf{F}(y)$ can be compared componentwise like real vectors. We denote this by

$$
\mathbf{F}(x) \preceq_{v} \mathbf{F}(y) \Leftrightarrow \mathbf{F}_{\mathbf{C}_{v}^{k_{i}}}^{i}(x) \preceq \mathbf{F}_{\mathbf{C}_{v}^{k_{i}}}^{i}(y) \forall i \in \Lambda_{m}
$$

A partial ordering can not compare all intervals. Due to the complexities associated with partial orderings, involved at different stages of $(M I O P)$, it is difficult to derive the efficient solution of $(M I O P)$ directly like general vector optimization problem. To avoid these complications, $(M I O P)$ is transformed to a general optimization problem in the subsequent sections. Some gradient free and gradient based results are established to study the existence of efficient solution of (MIOP) through the solution of the transformed problem. We accept the partial ordering $\preceq$ as defined in (2.2) to prove these results and call an efficient solution of (MIOP)

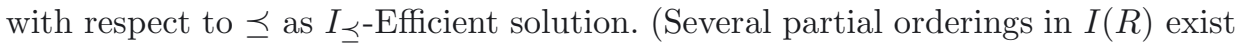
in literature (see $[5,7])$. The results of this paper are based on the partial ordering in the parametric form. However, similar theory may be developed with respect to

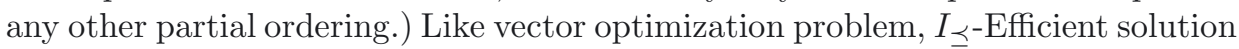
and properly $I_{\preceq}-$ Efficient solution of $(M I O P)$ may be defined as follows.

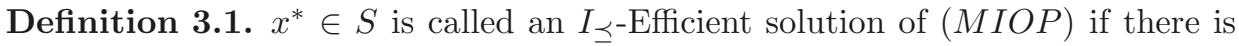
no $x \in S$ with

$$
\mathbf{F}_{\mathbf{C}_{v}^{k_{i}}}^{i}(x) \preceq \mathbf{F}_{\mathbf{C}_{v}^{k_{i}}}^{i}\left(x^{*}\right), i \in \Lambda_{m} \text { and for at least one } j \neq i, \mathbf{F}_{\mathbf{C}_{v}^{k_{j}}}^{j}(x) \prec \mathbf{F}_{\mathbf{C}_{v}^{j}}^{j}\left(x^{*}\right)
$$

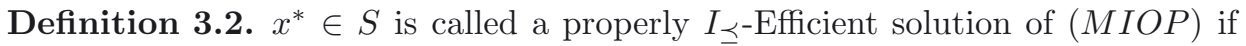

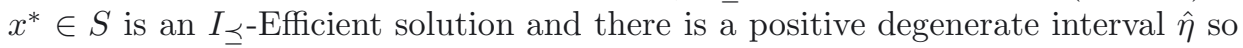
that for some $\bar{i} \in \Lambda_{m}$ and for every $x \in S$ with $\mathbf{F}_{\mathbf{C}_{v}^{k_{i}}}^{i}(x) \prec \mathbf{F}_{\mathbf{C}_{v}^{k_{i}}}^{i}\left(x^{*}\right)$, at least one $j \neq i$ exists with $\mathbf{F}_{\mathbf{C}_{v}^{k_{j}}}^{j}\left(x^{*}\right) \prec \mathbf{F}_{\mathbf{C}_{v}^{k_{j}}}^{j}(x)$ and

$$
\frac{\mathbf{F}_{\mathbf{C}_{v}^{k_{i}}}^{i}\left(x^{*}\right) \ominus \mathbf{F}_{\mathbf{C}_{v}^{k_{i}}}^{i}(x)}{\mathbf{F}_{\mathbf{C}_{v}^{j}}^{k_{j}}(x) \ominus \mathbf{F}_{\mathbf{C}_{v}^{k_{j}}}\left(x^{*}\right)} \preceq \hat{\eta} .
$$

Example 2. Consider the problem $\min _{\left(x_{1}, x_{2}\right) \in S}\left\{F_{1}\left(x_{1}, x_{2}\right), F_{2}\left(x_{1}, x_{2}\right)\right\}$, where $F_{1}\left(x_{1}, x_{2}\right)=[1,2] x_{1} \oplus[1,3], F_{2}\left(x_{1}, x_{2}\right)=[1,3] x_{2} \oplus[1,2]$ and $S=\left\{\left(x_{1}, x_{2}\right) \in\right.$ $\left.R^{n} \mid x_{1}^{2}+x_{2}^{2} \leq 1\right\}$.

$\left(x_{1}^{*}, x_{2}^{*}\right)=\left(-\frac{\sqrt{3}}{2},-\frac{1}{2}\right)$ is properly $I_{\preceq}$-Efficient solution of $(M I O P)$.

$F_{1}\left(x_{1}^{*}, x_{2}^{*}\right)=\left[1-\sqrt{3}, 3-\frac{\sqrt{3}}{2}\right], F_{2}\left(x_{1}^{*}, x_{2}^{*}\right)=\left[-\frac{1}{2}, \frac{3}{2}\right]$. Any point of the set $S$ is $\left(x_{1}^{n}, x_{2}^{n}\right)=\left(-\frac{\sqrt{2 n-1}}{n},-1+\frac{1}{n}\right), n \in N$. Substituting $\left(x_{1}^{n}, x_{2}^{n}\right)$ in place of $x$, the 
interval inequalities (3.7) becomes

$$
F_{1}\left(x_{1}^{n}, x_{2}^{n}\right) \preceq\left[1-\sqrt{3}, 3-\frac{\sqrt{3}}{2}\right] \text { and } F_{2}\left(x_{1}^{n}, x_{2}^{n}\right) \prec\left[-\frac{1}{2}, \frac{3}{2}\right] .
$$

Using the partial ordering $\preceq$ and $\prec$ stated in (2.2) and interval operations stated in $(2.1)$, (3.9) reduces to the following system of real inequalities.

$$
\frac{\sqrt{2 n-1}}{n} \geq \frac{\sqrt{3}}{2} \quad \text { and } \quad n>2, n \in N
$$

The system (3.10) has no solution. Consequently (3.7) has no solution. Hence by Definition $3.1,\left(-\frac{\sqrt{3}}{2},-\frac{1}{2}\right)$ is an $I_{\preceq}$-Efficient solution of $(M I O P)$.

Again

$$
\frac{F_{1}\left(x_{1}^{*}, x_{2}^{*}\right) \ominus F_{1}\left(x_{1}^{n}, x_{2}^{n}\right)}{F_{2}\left(x_{1}^{n}, x_{2}^{n}\right) \ominus F_{2}\left(x_{1}^{*}, x_{2}^{*}\right)}=\left[\frac{-2-\sqrt{3}+\frac{1}{n} \sqrt{2 n-1}}{\frac{3}{2}+\frac{3}{n}}, \frac{2-\frac{\sqrt{3}}{2}+\frac{2}{n} \sqrt{2 n-1}}{\frac{1}{n}-\frac{7}{2}}\right] .
$$

For a sequence of intervals $\left\{\left[a_{n}^{L}, a_{n}^{R}\right]\right\}, \lim _{n \rightarrow \infty}\left[a_{n}^{L}, a_{n}^{R}\right]=\left[\lim _{n \rightarrow \infty} a_{n}^{L}, \lim _{n \rightarrow \infty} a_{n}^{R}\right]$ (see [12]). Hence

$$
\lim _{n \rightarrow \infty} \frac{F_{1}\left(x_{1}^{*}, x_{2}^{*}\right) \ominus F_{1}\left(x_{1}^{n}, x_{2}^{n}\right)}{F_{2}\left(x_{1}^{n}, x_{2}^{n}\right) \ominus F_{2}\left(x_{1}^{*}, x_{2}^{*}\right)}=\left[\frac{-4-2 \sqrt{3}}{3}, \frac{\sqrt{3}-4}{7}\right] .
$$

Consider $\hat{\eta}=\left[\frac{4-2 \sqrt{3}}{3}, \frac{4-2 \sqrt{3}}{3}\right]$. Then we can write $\frac{F_{1}\left(x_{1}^{*}, x_{2}^{*}\right) \ominus F_{1}\left(x_{1}^{n}, x_{2}^{n}\right)}{F_{2}\left(x_{1}^{n}, x_{2}^{n}\right) \ominus F_{2}\left(x_{1}^{*}, x_{2}^{*}\right)} \preceq \hat{\eta}$. From

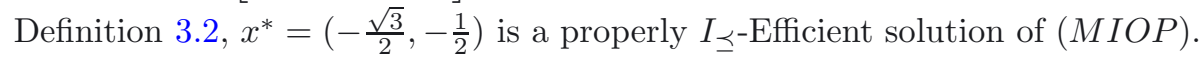

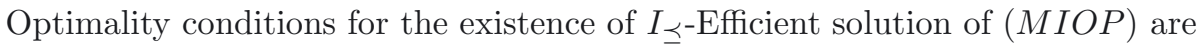
established in the following subsections.

\subsection{Optimality CONDition Without using DeRIVATive}

As discussed earlier, it is difficult to find the efficient solution of $(M I O P)$ directly due to the complexities arising in the partial ordering in the set of intervals. To address this difficulty, we construct a deterministic form of (MIOP) using some transformations as follows and prove that an optimal solution of the transformed

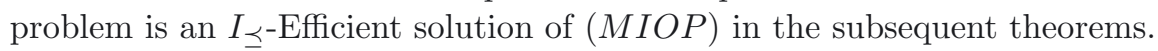

Consider a vector valued weight function $w$ (or $w(t))=$ $\left(w_{1}\left(t_{1}\right), w_{2}\left(t_{2}\right) \ldots, w_{m}\left(t_{m}\right)\right)^{T}, w_{i}\left(t_{i}\right)>0$, and $\mu_{i}>0, i \in \Lambda_{m}$, and construct an optimization problem

$$
\left(M I O P_{w}^{\mu}\right) \quad: \min _{x \in S} \Psi(x),
$$

where $\Psi(x)=\sum_{i \in \Lambda_{m}} \mu_{i} \psi_{i}(x)$, and $\psi_{i}(x)=\int_{k_{i}} w_{i}\left(t_{i}\right) f_{c_{i}\left(t_{i}\right)}^{i}(x) \mathrm{d} t_{i}, \mathrm{~d} t_{i}=$ $\mathrm{d} t_{i}^{1} \mathrm{~d} t_{i}^{2} \ldots \mathrm{d} t_{i}^{k_{i}}$ and $\int_{k_{i}}=\underbrace{\int_{0}^{1} \int_{0}^{1} \ldots \int_{0}^{1}}_{\left(k_{i} \text { times }\right)}$. 
Note. $w_{i}\left(t_{i}\right)$ may be treated as a preference weight function, which has to be provided by the decision maker. Different preference functions can be provided to estimate the Pareto optimal value of the model. For every $i, w_{i}\left(t_{i}\right)=1$ indicates that the investor's natural attitude is to estimate the mean. If $\int_{0}^{1} w_{i}\left(t_{i}\right) \mathrm{d} t_{i}=1$ for every $i$ then the investor's inclination is to estimate in between the optimistic and pessimistic optimal value. In the subsequent results we will see that any selection

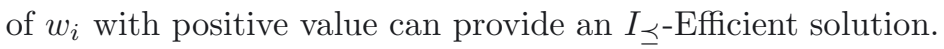

Theorem 3.3. If $x^{*} \in S$ is an optimal solution of $\left(M I O P_{w}^{\mu}\right)$ for some $w>_{v} 0$ and $\mu>_{v} 0$, then $x^{*}$ is an $I_{\preceq}$-Efficient solution of $(M I O P)$.

Proof. Let $x^{*} \in S$ be an optimal solution of $\left(M I O P_{w}^{\mu}\right), w>_{v} 0$ and $\mu>_{v} 0$. Assume

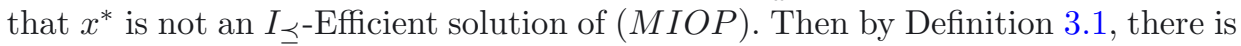
some $x \in S$ satisfying (3.7). Using (2.2), the inequalities in (3.7) can be rewritten as follows.

For some $x$ in $S$ and each $t_{i} \in[0,1]^{k_{i}}$, $f_{c_{i}\left(t_{i}\right)}^{i}(x) \leq f_{c_{i}\left(t_{i}\right)}^{i}\left(x^{*}\right), i \in \Lambda_{m}$ and $f_{c_{j}\left(t_{j}\right)}^{j}(x)<f_{c_{j}\left(t_{j}\right)}^{j}\left(x^{*}\right)$ for at least one $j \neq i$.

Since $w_{i}\left(t_{i}\right)>0$ and $\mu_{i}>0$, the above relations imply that $\sum_{i \in \Lambda_{m}} \mu_{i} \psi_{i}(x)<$ $\sum_{i \in \Lambda_{m}} \mu_{i} \psi_{i}\left(x^{*}\right)$. This is equivalent to $\Psi(x)<\Psi\left(x^{*}\right)$, which is impossible since

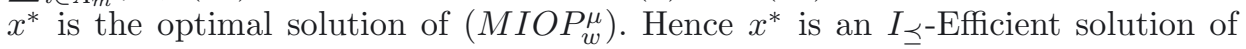
$(M I O P)$.

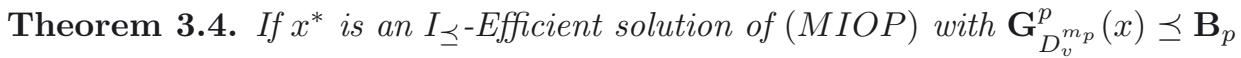
and $\mathbf{F}_{\mathbf{C}_{v}^{k_{i}}}^{i}, \mathbf{G}_{D_{v}^{m_{p}}}^{p}, p \in \Lambda_{q}, i \in \Lambda_{m}$ are interval valued convex functions with respect to $\preceq$ then there exists a weight function $w \geqq_{v} 0$ such that $x^{*}$ is an optimal solution of $\left(M I O P_{w}^{\mu}\right)$ for any $\mu>_{v} 0$.

Proof. Here $S=\left\{x \in R^{n} \mid \mathbf{G}_{D_{v}^{m_{p}}}^{p}(x) \preceq \mathbf{B}_{p}, p \in \Lambda_{q}\right\}=\bigcap_{p \in \Lambda_{q}}\left\{x \in R^{n} \mid g_{d_{p}\left(t_{p}^{\prime}\right)}^{p}(x) \leq\right.$ $\left.b_{p}\left(t_{p}^{\prime}\right), t_{p}^{\prime} \in[0,1]\right\}$. Since $\mathbf{G}_{D_{v}^{m_{p}}}^{p}$ is an interval valued convex function, so by Remark $2.2, g_{d_{p}\left(t_{p}^{\prime}\right)}^{p}(x)$ is a convex function. Hence $S$ is a convex set. Suppose $x^{*} \in S$

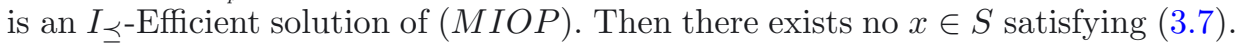
From Remark 2.2, we can conclude that $f_{c_{i}\left(t_{i}\right)}^{i}$ is convex on a convex set $S$ for each $t_{i}$. Since (3.7) has no solution, so using the concept of partial ordering and interval valued function in Section 2 , we can conclude that, for every $t_{i}$ in $[0,1]^{k_{i}}$, the following system has no solution on $S$.

$$
\begin{array}{r}
f_{c_{i}\left(t_{i}\right)}^{i}(x)-f_{c_{i}\left(t_{i}\right)}^{i}\left(x^{*}\right) \leq 0 \forall i \in \Lambda_{m} \\
\text { and } f_{c_{j}\left(t_{j}\right)}^{j}(x)-f_{c_{j}\left(t_{j}\right)}^{j}\left(x^{*}\right)<0 \\
\text { for at least one } j \neq i
\end{array}
$$

If we denote $F(x, t)=\left(f_{c_{1}\left(t_{1}\right)}^{1}(x)-f_{c_{1}\left(t_{1}\right)}^{1}\left(x^{*}\right), \ldots, f_{c_{m}\left(t_{m}\right)}^{m}(x)-f_{c_{m}\left(t_{m}\right)}^{m}\left(x^{*}\right)\right)^{T}$ then above system implies that $F(x, t) \leqq_{v} 0$ has no solution for every $t$. This implies that $F(x, t)<_{v} 0$ has no solution for every $t$. Hence from Proposition 2.3, there 
exists a real vector $u=\left(u_{1}, u_{2}, \ldots, u_{m}\right)^{T}, u \geqq v 0$ such that $u^{T} F(x, t) \geq 0$ is true for all $x \in S$. Define $w_{i}:[0,1]^{k_{i}} \rightarrow R_{+} \cup\{0\}$ by $w_{i}\left(t_{i}\right)=u_{i}, i \in \Lambda_{m}$. Then $u^{T} F(x, t) \geq 0$ is same as

$$
w(t)^{T} F(x) \geq 0, \forall x \in S
$$

This implies that $\sum_{i \in \Lambda_{m}} w_{i}\left(t_{i}\right) f_{c_{i}\left(t_{i}\right)}^{i}(x) \geq \sum_{i \in \Lambda_{m}} w_{i}\left(t_{i}\right) f_{c_{i}\left(t_{i}\right)}^{i}\left(x^{*}\right) \forall x \in S$. Hence for $\mu_{i}>0, \sum_{i \in \Lambda_{m}} \mu_{i} \psi_{i}(x) \geq \sum_{i \in \Lambda_{m}} \mu_{i} \psi_{i}\left(x^{*}\right) \forall x \in S$. This is equivalent to $\Psi(x) \geq$ $\Psi\left(x^{*}\right) \forall x \in S$, which implies that $x^{*}$ is an optimal solution of $\left(M I O P_{w}^{\mu}\right)$ for $w \geqq v$, $\mu>_{v} 0$.

Theorem 3.5. If $x^{*} \in S$ is an optimal solution of $\left(M I O P_{w}^{\mu}\right), w>_{v} 0, w_{i}$ are continuous functions satisfying $\int_{k_{i}} w_{i}\left(t_{i}\right) \mathrm{d} t_{i}=1$ and $\mu>_{v} 0$ then $x^{*}$ is a properly

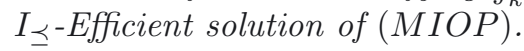

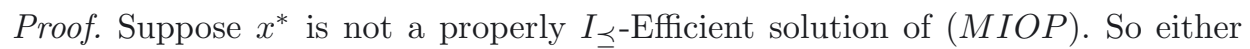

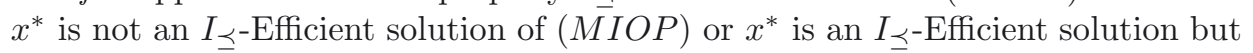
does not satisfy the conditions in (3.8).

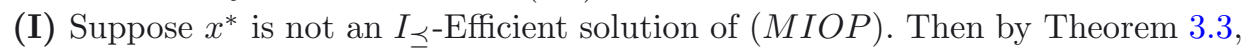
$x^{*}$ is not an optimal solution of $\left(M_{I O P_{w}^{\mu}}\right)$ for any choice of $w>_{v} 0$ and $\mu>_{v} 0$, which contradicts that $x^{*}$ is the optimal solution of $\left(M I O P_{w}^{\mu}\right)$.

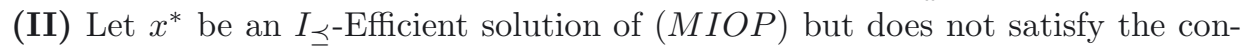

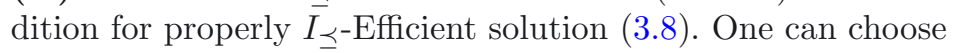

$$
\eta=(m-1) \max _{i, j} \max _{t_{i}, \bar{t}_{i}, t_{j}, \bar{t}_{j}}\left\{\frac{\mu_{j} w_{j}\left(t_{j}\right) w_{j}\left(\bar{t}_{j}\right)}{\mu_{i} w_{i}\left(t_{i}\right) w_{i}\left(\bar{t}_{i}\right)}\right\} \text { for } m \geq 2, i \neq j, t_{i}, \bar{t}_{i} \in[0,1]^{k_{i}}
$$

Since $w_{i}$ is continuous so $\eta$ exists. Then from Definition 3.2, for some $i \in \Lambda_{m}$ and some $x \in S$ with $\mathbf{F}_{\mathbf{C}_{v}^{k_{i}}}^{i}(x) \prec \mathbf{F}_{\mathbf{C}_{v}^{k_{i}}}^{i}\left(x^{*}\right)$,

$$
\frac{\mathbf{F}_{\mathbf{C}_{v}^{k_{i}}}^{i}\left(x^{*}\right) \ominus \mathbf{F}_{\mathbf{C}_{v}^{k_{i}}}^{i}(x)}{\mathbf{F}_{\mathbf{C}_{v}^{k_{j}}}^{k_{j}}(x) \ominus \mathbf{F}_{\mathbf{C}_{v}^{j}}^{k_{j}}\left(x^{*}\right)} \succ \hat{\eta}, \forall j \in \Lambda_{m}, i \neq j,
$$

with $\mathbf{F}_{\mathbf{C}_{v}^{k_{j}}}^{j}\left(x^{*}\right) \prec \mathbf{F}_{\mathbf{C}_{v}^{k_{j}}}^{j}(x)$ holds for $\hat{\eta}=[\eta, \eta]$.

(3.12) means for every $t_{i}, \bar{t}_{i}$,

$$
\frac{f_{c_{i}\left(t_{i}\right)}^{i}\left(x^{*}\right)-f_{c_{i}\left(\bar{t}_{i}\right)}^{i}(x)}{f_{c_{j}\left(t_{j}\right)}^{j}(x)-f_{c_{j}\left(\bar{t}_{j}\right)}^{j}\left(x^{*}\right)}>\eta \geq(m-1)\left\{\frac{\mu_{j} w_{j}\left(t_{j}\right) w_{j}\left(\bar{t}_{j}\right)}{\mu_{i} w_{i}\left(t_{i}\right) w_{i}\left(\bar{t}_{i}\right)}\right\} \forall j \in \Lambda_{m} /\{i\},
$$

which is

$$
\begin{array}{r}
\mu_{i} w_{i}\left(t_{i}\right) w_{i}\left(\bar{t}_{i}\right)\left(f_{c_{i}\left(t_{i}\right)}^{i}\left(x^{*}\right)-f_{c_{i}\left(\bar{t}_{i}\right)}^{i}(x)\right)> \\
(m-1) \mu_{j} w_{j}\left(t_{j}\right) w_{j}\left(\bar{t}_{j}\right)\left(f_{c_{j}\left(t_{j}\right)}^{j}(x)-f_{c_{j}\left(\bar{t}_{j}\right)}^{j}\left(x^{*}\right)\right)
\end{array}
$$


So

$$
\begin{aligned}
\mu_{i} \int_{k_{i}} \int_{k_{j}} w_{i}\left(t_{i}\right) w_{i}\left(\bar{t}_{i}\right)\left(f_{c_{i}\left(t_{i}\right)}^{i}\left(x^{*}\right)\right. & \left.-f_{c_{i}\left(\bar{t}_{i}\right)}^{i}(x)\right) \mathrm{d} t_{i} \mathrm{~d} t_{j}>(m-1) \mu_{j} \\
\times & \times \int_{k_{i}} \int_{k_{j}} w_{j}\left(t_{j}\right) w_{j}\left(\bar{t}_{j}\right)\left(f_{c_{j}\left(t_{j}\right)}^{j}(x)-f_{c_{j}\left(\bar{t}_{j}\right)}^{j}\left(x^{*}\right)\right) \mathrm{d} t_{i} \mathrm{~d} t_{j}
\end{aligned}
$$

Since $\int_{k_{i}} w_{i}\left(t_{i}\right) \mathrm{d} t_{i}=1$, after integrating the above inequality becomes

$$
\mu_{i}\left(\psi_{i}\left(x^{*}\right)-\psi_{i}(x)\right)>(m-1) \mu_{j}\left(\psi_{j}(x)-\psi_{j}\left(x^{*}\right)\right)
$$

Hence

$$
\sum_{j \in \Lambda_{m}, j \neq i} \mu_{i}\left(\psi_{i}\left(x^{*}\right)-\psi_{i}(x)\right)>(m-1) \sum_{j \in \Lambda_{m}, j \neq i} \mu_{j}\left(\psi_{j}(x)-\psi_{j}\left(x^{*}\right)\right)
$$

This implies $\mu_{i}\left(\psi_{i}\left(x^{*}\right)-\psi_{i}(x)\right)>\sum_{j \in \Lambda_{m}, j \neq i} \mu_{j}\left(\psi_{j}(x)-\psi_{j}\left(x^{*}\right)\right)$. Hence $\sum_{j \in \Lambda_{m}} \mu_{j} \psi_{j}\left(x^{*}\right)>\sum_{j \in \Lambda_{m}} \mu_{j} \psi_{j}(x)$.

That is, $\Psi\left(x^{*}\right)>\Psi(x)$. This contradicts the assumption that $x^{*}$ is the optimal solution of $\left(M I O P_{w}^{\mu}\right)$.

\subsection{Optimality CONDition Using DERIVATive}

For a feasible point $x^{*} \in S$, denote $J\left(x^{*}\right)=\left\{p: \mathbf{G}_{D_{v}^{m_{p}}}^{p}\left(x^{*}\right)=\mathbf{B}_{p}, p \in \Lambda_{q}\right\}$. That is, $J\left(x^{*}\right)=\left\{p: g_{d_{p}\left(t_{p}^{\prime}\right)}^{p}\left(x^{*}\right)=b_{p}\left(t_{p}^{\prime}\right), \forall t_{p}^{\prime} \in[0,1], p \in \Lambda_{q}\right\}$.

Theorem 3.6. Suppose $\mathbf{F}_{\mathbf{C}_{v}^{k_{i}}}^{i}$ and $\mathbf{G}_{D_{v}^{m_{p}}}^{p}$ are differentiable functions and convex with respect to $\preceq$ at $x^{*} \in S$ of $(M I O P)$, satisfying

$$
\sum_{i \in \Lambda_{m}} \mu_{i} \nabla \mathbf{F}_{\mathbf{C}_{v}^{k_{i}}}^{i}\left(x^{*}\right) \oplus \sum_{p \in J\left(x^{*}\right)} \nu_{p} \nabla \mathbf{G}_{D_{v}^{m_{p}}}^{p}\left(x^{*}\right)=\mathbf{0}
$$

where $\mu_{i}>0, i \in \Lambda_{m}$ and for every $p \in \Lambda_{q}, \nu_{p} \geq 0$ if $\mathbf{G}_{D_{v}^{m_{p}}}^{p}(x) \preceq \mathbf{B}_{p} ; \nu_{p} \leq 0$ if $\mathbf{G}_{D_{v}^{m_{p}}}^{p}(x) \succeq \mathbf{B}_{p}$; and $\nu_{p}$ is unrestricted if $p \in J\left(x^{*}\right)$. Then $x^{*}$ is an $I_{\preceq \text {-Efficient }}$ solution for $(M I O P)$.

Proof. Suppose $\mathbf{F}_{\mathbf{C}_{v}^{k_{i}}}^{i}$ and $\mathbf{G}_{D_{v}^{m_{p}}}^{p}$ are convex at $x^{*}$ with respect to $\preceq$. From Remark 2.2 it is true that $f_{c_{i}\left(t_{i}\right)}^{i}$ and $g_{d_{p}\left(t_{p}^{\prime}\right)}^{p}$ are convex functions at $x^{*}$ for every $t_{i}, t_{p}^{\prime}$. So for all $x \in S$ and all $t_{i}, t_{p}^{\prime}$,

$$
\begin{array}{r}
f_{c_{i}\left(t_{i}\right)}^{i}(x)-f_{c_{i}\left(t_{i}\right)}^{i}\left(x^{*}\right) \geq\left(x-x^{*}\right)^{T} \nabla f_{c_{i}\left(t_{i}\right)}^{i}\left(x^{*}\right), \forall i \in \Lambda_{m} \\
g_{d_{p}\left(t_{p}^{\prime}\right)}^{p}(x)-g_{d_{p}\left(t_{p}^{\prime}\right)}^{p}\left(x^{*}\right) \geq\left(x-x^{*}\right)^{T} \nabla g_{d_{p}\left(t_{p}^{\prime}\right)}^{p}\left(x^{*}\right), \forall p \in \Lambda_{q}
\end{array}
$$

From (2.3), Equation (3.13) becomes

$$
\sum_{i \in \Lambda_{m}} \mu_{i} \nabla f_{c_{i}\left(t_{i}\right)}^{i}\left(x^{*}\right)+\sum_{p \in J\left(x^{*}\right)} \nu_{p} \nabla g_{d_{p}\left(t_{p}^{\prime}\right)}^{p}\left(x^{*}\right)=0, \forall t_{i}, t_{p}^{\prime}
$$


For scalers $\mu_{i}>0, i \in \Lambda_{m}$,

$$
\begin{aligned}
\sum_{i \in \Lambda_{m}} \mu_{i}\left(f_{c_{i}\left(t_{i}\right)}^{i}(x)-f_{c_{i}\left(t_{i}\right)}^{i}\left(x^{*}\right)\right) & \geq \sum_{i \in \Lambda_{m}} \mu_{i}\left(x-x^{*}\right)^{T} \nabla f_{c_{i}\left(t_{i}\right)}^{i}\left(x^{*}\right) \quad(\text { from }(3.14)) \\
= & -\sum_{p \in J\left(x^{*}\right)} \nu_{p}\left(x-x^{*}\right)^{T} \nabla g_{d_{p}\left(t_{p}^{\prime}\right)}^{p}\left(x^{*}\right)(\text { from }(3.16)) \\
\geq & -\sum_{p \in J\left(x^{*}\right)} \nu_{p}\left(g_{d_{p}\left(t_{p}^{\prime}\right)}^{p}(x)-g_{d_{p}\left(t_{p}^{\prime}\right)}^{p}\left(x^{*}\right)\right)(\text { from }(3.15)) \\
= & -\sum_{p \in J\left(x^{*}\right)} \nu_{p}\left(g_{d_{p}\left(t_{p}^{\prime}\right)}^{p}(x)-b_{p}\left(t_{p}^{\prime}\right)\right. \\
& \left.-g_{d_{p}\left(t_{p}^{\prime}\right)}^{p}\left(x^{*}\right)+b_{p}\left(t_{p}^{\prime}\right)\right)
\end{aligned}
$$

From the definition of $\nu_{p}, \nu_{p}\left(g_{d_{p}\left(t_{p}^{\prime}\right)}^{p}(x)-b_{p}\left(t_{p}^{\prime}\right)\right) \leq 0, \forall x \neq x^{*}$ and for $p \in J\left(x^{*}\right)$, $\nu_{i}\left(g_{d_{p}\left(t_{p}^{\prime}\right)}^{p}\left(x^{*}\right)-b_{p}\left(t_{p}^{\prime}\right)\right)=0$. Hence the above inequality becomes

$$
\sum_{i \in \Lambda_{m}} \mu_{i} f_{c_{i}\left(t_{i}\right)}^{i}(x) \geq \sum_{i \in \Lambda_{m}} \mu_{i} f_{c_{i}\left(t_{i}\right)}^{i}\left(x^{*}\right) .
$$

Now for any $w>_{v} 0, \sum_{i \in \Lambda_{m}} \mu_{i} \psi_{i}(x) \geq \sum_{i \in \Lambda_{m}} \mu_{i} \psi_{i}\left(x^{*}\right)$. This is same as $\Psi(x) \geq$ $\Psi\left(x^{*}\right)$. That is, $x^{*}$ is an optimal solution for $\left(M I O P_{w}^{\mu}\right)$, for $w, \mu>_{v} 0$. Hence from

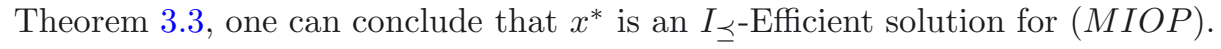

\subsection{NumericAl EXAMPLES}

Some results of Sections 3.1 and 3.2 are illustrated in the following examples. Example 3 justifies the application of Theorem 3.3 and Example 4 justifies the application of Theorem 3.6.

Example 3. Consider the following optimization problem whose objective functions and constraints are nonlinear interval valued functions.

$$
\begin{aligned}
(M I O P) & \min \quad\left\{[-2,0] x_{1},[-1,2] x_{1} \oplus[1,1] x_{2}^{2}\right\} \\
\text { subject to } & {[0.5,1.5] x_{1} \oplus[1.5,2.5] x_{2} \preceq[2.5,3.5], } \\
& {[0.5,1.5] x_{1}^{2} \oplus[1,1] x_{2} \preceq[0.05,0.2], } \\
& x_{1} \geq 0, x_{2} \geq 0 .
\end{aligned}
$$

Denote $\mathbf{F}_{\mathbf{C}_{v}^{1}}^{1}\left(x_{1}, x_{2}\right)=[-2,0] x_{1}, \mathbf{F}_{\mathbf{C}_{v}^{2}}^{2}\left(x_{1}, x_{2}\right)=[-1,2] x_{1} \oplus[1,1] x_{2}^{2}, \mathbf{G}_{D_{v}^{2}}^{1}\left(x_{1}, x_{2}\right)=$ $[0.5,1.5] x_{1} \oplus[1.5,2.5] x_{2}, \mathbf{G}_{D_{v}^{2}}^{2}\left(x_{1}, x_{2}\right)=[0.5,1.5] x_{1}^{2} \oplus[1,1] x_{2}$.

Then $f_{c_{1}\left(t_{1}\right)}^{1}\left(x_{1}, x_{2}\right)=\left(-2+2 t_{1}\right) x_{1}$ and $f_{c_{2}\left(t_{2}\right)}^{2}\left(x_{1}, x_{2}\right)=\left(-1+3 t_{2}\right) x_{1}+x_{2}^{2}$, where $t_{1}, t_{2} \in[0,1]$. Consider the weight functions $w_{1}\left(t_{1}\right)=1+t_{1}, w_{2}\left(t_{2}\right)=$ $\left(1+t_{2}\right)^{2}$ and $\mu_{1}=\frac{3}{4}, \mu_{2}=\frac{1}{4}$. Then $\psi_{1}\left(x_{1}, x_{2}\right)=\int_{0}^{1} w_{1}\left(t_{1}\right) f_{c_{1}\left(t_{1}\right)}^{1}\left(x_{1}, x_{2}\right) \mathrm{d} t_{1}=$ $-\frac{4}{3} x_{1}$ and $\psi_{2}\left(x_{1}, x_{2}\right)=\int_{0}^{1} w_{2}\left(t_{2}\right) f_{c_{2}\left(t_{2}\right)}^{2}\left(x_{1}, x_{2}\right) \mathrm{d} t_{2}=\frac{23}{12} x_{1}+\frac{7}{3} x_{2}^{2}$. Using (3.6), the 
parametric form of $\mathbf{G}_{D_{v}^{2}}^{1}\left(x_{1}, x_{2}\right) \preceq[2.5,3.5]$ and $\mathbf{G}_{D_{v}^{2}}^{2}\left(x_{1}, x_{2}\right) \preceq[0.05,0.2]$ can be written as $g_{d_{1}\left(t_{1}^{\prime}\right)}^{1}\left(x_{1}, x_{2}\right) \leq\left(2.5+t_{1}^{\prime}\right) \forall t_{1}^{\prime} \in[0,1]$ and $g_{d_{2}\left(t_{2}^{\prime}\right)}^{2}\left(x_{1}, x_{2}\right) \leq(0.05+$ $\left.0.15 t_{2}^{\prime}\right) \forall t_{2}^{\prime} \in[0,1]$, respectively, where

$$
g_{d_{1}\left(t_{1}^{\prime}\right)}^{1}\left(x_{1}, x_{2}\right)=\left(0.5+t_{1}^{\prime}\right) x_{1}+\left(1.5+t_{1}^{\prime}\right) x_{2}, g_{d_{2}\left(t_{2}^{\prime}\right)}^{2}\left(x_{1}, x_{2}\right)=\left(0.5+t_{2}^{\prime}\right) x_{1}^{2}+x_{2} .
$$

Hence

$$
\begin{aligned}
& S=\left\{\left(x_{1}, x_{2}\right) \in R^{2} \mid g_{d_{1}\left(t_{1}^{\prime}\right)}^{1}\left(x_{1}, x_{2}\right) \leq\left(2.5+t_{1}^{\prime}\right), g_{d_{2}\left(t_{2}^{\prime}\right)}^{2}\left(x_{1}, x_{2}\right) \leq\left(0.05+0.15 t_{2}^{\prime}\right) ;\right. \\
&\left.t_{1}^{\prime}, t_{2}^{\prime} \in[0,1], x_{1} \geq 0, x_{2} \geq 0\right\} \\
&=\left\{\left(x_{1}, x_{2}\right) \in R^{2} \mid 0.5 x_{1}+1.5 x_{2} \leq 2.5,1.5 x_{1}+2.5 x_{2} \leq 3.5,0.5 x_{1}^{2}+x_{2} \leq 0.05,\right. \\
&\left.1.5 x_{1}^{2}+x_{2} \leq 0.2, x_{1} \geq 0, x_{2} \geq 0\right\} .
\end{aligned}
$$

The deterministic problem corresponding to $(M I O P)$ becomes

$$
\left(M I O P_{w}^{\mu}\right): \min _{\left(x_{1}, x_{2}\right) \in S}-\frac{25}{48} x_{1}+\frac{7}{12} x_{2}^{2}
$$

Using LINGO the optimal solution of the above problem is found as $(0.3162278,0)$.

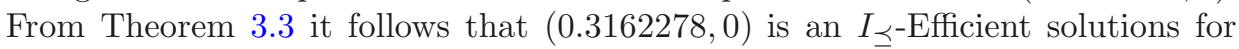
$(M I O P)$.

This may also be verified using Definition 3.1.

Example 4. Consider the following optimization problem whose objective functions and constraints are nonlinear interval valued convex functions with respect to $\preceq$.

$$
\begin{gathered}
\min \left\{[1,2] x_{1}^{2} \oplus[2,3] x_{2}^{2} \oplus[1,2],[2,4] x_{1}^{2} \oplus[4,6] x_{2}^{2} \oplus[3,5]\right\} \\
\text { subject to }[1,6] x_{1} \oplus[1,2] x_{2} \succeq[1,12],[1,2] x_{1} \oplus\left[\frac{2}{3}, 1\right] x_{2} \succeq\left[\frac{11}{5}, \frac{21}{5}\right] .
\end{gathered}
$$

Denote $\mathbf{F}_{\mathbf{C}_{v}^{3}}^{1}\left(x_{1}, x_{2}\right)=[1,2] x_{1}^{2} \oplus[2,3] x_{2}^{2} \oplus[1,2], \mathbf{F}_{\mathbf{C}_{v}^{3}}^{2}\left(x_{1}, x_{2}\right)=[2,4] x_{1}^{2} \oplus[4,6] x_{2}^{2} \oplus$ $[3,5]$,

$\mathbf{G}_{\mathbf{D}_{v}^{2}}^{1}\left(x_{1}, x_{2}\right)=[1,6] x_{1} \oplus[1,2] x_{2}$ and $\mathbf{G}_{\mathbf{D}_{v}^{2}}^{2}\left(x_{1}, x_{2}\right)=[1,2] x_{1} \oplus\left[\frac{2}{3}, 1\right] x_{2}$.

Then $f_{c_{1}\left(t_{1}\right)}^{1}\left(x_{1}, x_{2}\right)=\left(1+t_{1}^{1}\right) x_{1}^{2}+\left(2+t_{1}^{2}\right) x_{2}^{2}+\left(1+t_{1}^{3}\right)$ and $f_{c_{2}\left(t_{2}\right)}^{2}\left(x_{1}, x_{2}\right)=$ $\left(2+2 t_{2}^{1}\right) x_{1}^{2}+\left(4+2 t_{2}^{2}\right) x_{2}^{2}+\left(3+2 t_{2}^{3}\right)$, where $t_{1}^{j}, t_{2}^{j} \in[0,1], j=1,2,3$.

Using (3.6), the parametric form of $\mathbf{G}_{D_{v}^{2}}^{1}\left(x_{1}, x_{2}\right) \succeq[1,12]$ and $\mathbf{G}_{D_{v}^{2}}^{2}\left(x_{1}, x_{2}\right) \succeq$ $\left[\frac{11}{5}, \frac{21}{5}\right]$ can be written as

$g_{d_{1}\left(t_{1}^{\prime}\right)}^{1}\left(x_{1}, x_{2}\right) \geq\left(1+11 t_{1}^{\prime}\right) \forall t_{1}^{\prime} \in[0,1]$ and $g_{d_{2}\left(t_{2}^{\prime}\right)}^{2}\left(x_{1}, x_{2}\right) \geq\left(\frac{11}{5}+2 t_{2}^{\prime}\right) \forall t_{2}^{\prime} \in[0,1]$,

where $g_{d_{1}\left(t_{1}^{\prime}\right)}^{1}\left(x_{1}, x_{2}\right)=\left(1+5 t_{1}^{\prime}\right) x_{1}+\left(1+t_{1}^{\prime}\right) x_{2}, g_{d_{2}\left(t_{2}^{\prime}\right)}^{2}\left(x_{1}, x_{2}\right)=\left(1+t_{2}^{\prime}\right) x_{1}+$ $\left(\frac{2}{3}+\frac{1}{3} t_{2}^{\prime}\right) x_{2}$. Following the procedure of Example 3 with $w_{1}\left(t_{1}^{1}, t_{1}^{2}, t_{1}^{3}\right)=1+t_{1}^{1} t_{1}^{2}$ 
and $w_{2}\left(t_{2}^{1}, t_{2}^{2}, t_{2}^{3}\right)=1+t_{2}^{1} t_{2}^{2} t_{2}^{3}$ and $\mu_{1}=\frac{1}{2}, \mu_{2}=\frac{1}{2}$, we get an $I_{\preceq \text {-Efficient solution }}$ of this problem as $x^{*}=\left(x_{1}^{*}, x_{2}^{*}\right)=\left(\frac{9}{5}, \frac{3}{5}\right)$.

Since $f_{c_{1}\left(t_{1}\right)}^{1}, f_{c_{2}\left(t_{2}\right)}^{2}, g_{d_{1}\left(t_{1}^{\prime}\right)}^{1}$ and $g_{d_{2}\left(t_{2}^{\prime}\right)}^{2}$ are convex in $R^{2}$ for every $t_{1}, t_{2}, t_{1}^{\prime}, t_{2}^{\prime}$, so from Remark 2.2 , the interval valued functions $\mathbf{F}_{\mathbf{C}_{v}^{3}}^{1}, \mathbf{F}_{\mathbf{C}_{v}^{3}}^{2}, \mathbf{G}_{\mathbf{D}_{v}^{2}}^{1}$ and $\mathbf{G}_{\mathbf{D}_{v}^{2}}^{2}$ are convex functions with respect to $\preceq$. Here $J\left(x^{*}\right)=\{2\}$.

$$
\begin{aligned}
\nabla \mathbf{F}_{\mathbf{C}_{v}^{3}}^{1}\left(x_{1}^{*}, x_{2}^{*}\right)= & \left(\begin{array}{l}
\left.\left[\min _{t_{1} \in[0,1]^{3}} \frac{\partial f_{c_{1}\left(t_{1}\right)}^{1}\left(x_{1}^{*}, x_{2}^{*}\right)}{\partial x_{1}}, \max _{t_{1} \in[0,1]^{3}} \frac{\partial f_{c_{1}\left(t_{1}\right)}^{1}\left(x_{1}^{*}, x_{2}^{*}\right)}{\partial x_{1}}\right]\right) \\
{\left[\min _{t_{1} \in[0,1]^{3}} \frac{\partial f_{c_{1}\left(t_{1}\right)}^{1}\left(x_{1}^{*}, x_{2}^{*}\right)}{\partial x_{2}}, \max _{t_{1} \in[0,1]^{3}} \frac{\partial f_{c_{1}\left(t_{1}\right)}^{1}\left(x_{1}^{*}, x_{2}^{*}\right)}{\partial x_{2}}\right]}
\end{array}\right) \\
= & \left(\begin{array}{l}
{\left[\min _{t_{1}^{1} \in[0,1]}\left(1+t_{1}^{1}\right) \frac{18}{5}, \max _{t_{1}^{1} \in[0,1]}\left(1+t_{1}^{1}\right) \frac{18}{5}\right]} \\
{\left[\min _{t_{1}^{2} \in[0,1]}\left(2+t_{1}^{2}\right) \frac{6}{5}, \max _{t_{1}^{2} \in[0,1]}\left(2+t_{1}^{2}\right) \frac{6}{5}\right]}
\end{array}\right)=\left(\begin{array}{c}
{\left[\frac{18}{5}, \frac{36}{5}\right]} \\
{\left[\frac{12}{5}, \frac{18}{5}\right]}
\end{array}\right) .
\end{aligned}
$$

Similarly, $\nabla \mathbf{F}_{\mathbf{C}_{v}^{3}}^{2}\left(x_{1}^{*}, x_{2}^{*}\right)=\left(\begin{array}{c}{\left[\frac{36}{5}, \frac{72}{5}\right]} \\ {\left[\frac{24}{5}, \frac{36}{5}\right]}\end{array}\right), \nabla \mathbf{G}_{\mathbf{D}_{v}^{2}}^{2}\left(x_{1}^{*}, x_{2}^{*}\right)=\left(\begin{array}{c}{[1,2]} \\ {\left[\frac{2}{3}, 1\right]}\end{array}\right)$.

Equation (3.13) is equivalent to

$$
\begin{aligned}
& \mu_{1} \nabla \mathbf{F}_{\mathbf{C}_{v}^{3}}^{1}\left(x_{1}^{*}, x_{2}^{*}\right) \oplus \mu_{2} \nabla \mathbf{F}_{\mathbf{C}_{v}^{3}}^{2}\left(x_{1}^{*}, x_{2}^{*}\right) \oplus \nu_{1} \nabla \mathbf{G}_{\mathbf{D}_{v}^{2}}^{2}\left(x_{1}^{*}, x_{2}^{*}\right)=\mathbf{0} \\
\equiv & \mu_{1}\left(\begin{array}{c}
{\left[\frac{18}{5}, \frac{36}{5}\right]} \\
{\left[\frac{12}{5}, \frac{18}{5}\right]}
\end{array}\right) \oplus \mu_{2}\left(\begin{array}{c}
{\left[\frac{36}{5}, \frac{72}{5}\right]} \\
{\left[\frac{24}{5}, \frac{36}{5}\right]}
\end{array}\right) \oplus \nu_{1}\left(\begin{array}{c}
{[1,2]} \\
{\left[\frac{2}{3}, 1\right]}
\end{array}\right)=\left(\begin{array}{c}
{[0,0]} \\
{[0,0]}
\end{array}\right) \\
\equiv & \mu_{1}\left[\frac{18}{5}, \frac{36}{5}\right] \oplus \mu_{2}\left[\frac{36}{5}, \frac{72}{5}\right] \oplus \nu_{1}[1,2]=[0,0], \\
& \text { and } \mu_{1}\left[\frac{12}{5}, \frac{18}{5}\right] \oplus \mu_{2}\left[\frac{24}{5}, \frac{36}{5}\right] \oplus \nu_{1}\left[\frac{2}{3}, 1\right]=[0,0] \\
\equiv & \frac{18}{5} \mu_{1}+\frac{36}{5} \mu_{2}+\nu_{1}=0, \text { and } \frac{12}{5} \mu_{1}+\frac{24}{5} \mu_{2}+\frac{2}{3} \nu_{1}=0
\end{aligned}
$$

$\mu_{1}=\frac{1}{2}, \mu_{2}=\frac{1}{2}, \nu_{1}=-\frac{27}{5}$ satisfy (3.17). Hence by Theorem 3.6, $\left(\frac{9}{5}, \frac{3}{5}\right)$ is an

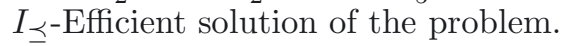

\section{Conclusion}

In this paper, a multi-objective interval optimization problem is solved after transforming it to a general deterministic optimization problem, which is free from interval uncertainty. Relation between the solution of original problem and the transformed problem is established under some assumptions. As we discussed earlier, different type of nonlinear multi-objective interval optimization problems are studied by Dunwei Gong et al. [2], Soares et al. [10] and Wu [13]. Dunwei Gong et al. and Soares et al. have suggested some evolutionary algorithms for MIOP but they have not studied the conditions for the existence of solution of MIOP. Wu has studied the conditions for the existence of solution of a particular type of $M I O P$ whose objective functions are interval valued functions but all constraints 
are real valued functions. This paper discusses the conditions for the existence of solution of MIOP model whose objective functions as well as constraints are nonlinear interval valued functions. This may be treated as an advantage of this paper. While transforming the original model to a deterministic form, a weight function is required, which has to be provided by the decision maker. Sometimes selection of the weight function may create a burden to the decision maker, but choice of the weight function has no effect on the theoretical developments. Proofs of the results of this paper require a partial ordering in parametric form. Any other partial ordering can also be used to develop similar methodologies in the light of present development. However in case of a different partial ordering the formulation of the transformed problem and the proof of the theorems may differ. Duality theory plays an important role for the existence of solution of a general multi-objective interval optimization problem. In the light of this methodology duality theory for a general multi-objective interval optimization problem can be established which is the future scope of the present work.

Acknowledgements. The authors thank two anonymous referees whose justified critical remarks on the original version led to an essential reworking of the paper.

\section{REFERENCES}

[1] A. Bhurjee and G. Panda, Efficient solution of interval optimization problem. Math. Meth. Oper. Res. 76 (2012) 273-288.

[2] D. Gong, J. Sun and X. Ji, Evolutionary algorithms with preference polyhedron for interval multi-objective optimization problems. Inform. Sci. 233 (2013) 141-161.

[3] M. Hladik, Computing the tolerances in multiobjective linear programming. Optim. Methods Softw. 23 (2008) 731-739.

[4] M. Inuiguchi and M. Sakawa, Possible and necessary efficiency in possibilistic multiobjective linear programming problems and possible efficiency test. Fuzzy Sets Syst. 78 (1996) 231241.

[5] H. Ishibuchi and H. Tanaka, Multiobjective programming in optimization of the interval objective function. Eur. J. Oper. Res. 48 (1990) 219-225.

[6] O. Mangasarian, Nonlinear Programming. New York: McGraw Hill (1969).

[7] R. Moore, Interval Analysis. Prentice-Hall (1966).

[8] C. Oliveira and C.H. Antunes, Multiple objective linear programming models with interval coefficients an illustrated overview. Eur. J. Oper. Res. 181 (2007) 1434-1463.

[9] S. Rivaz and M. Yaghoobi, Minimax regret solution to multiobjective linear programming problems with interval objective functions coefficients. Central Eur. J. Oper. Res. 21 (2013) 625-649.

[10] G. Soares, R. Parreiras, L. Jaulin, J. Vasconcelos and C. Maia, Interval robust multiobjective algorithm. Nonlinear Anal. Theor. Meth. Appl. 71 (2009) 1818-1825.

[11] B. Urli and R. Nadeau, An interactive method to multiobjective linear programming problem with interval coefficients. INFOR 30 (1992) 127-137.

[12] H.C. Wu, On interval-valued nonlinear programming problems. J. Math. Anal. Appl. 338 (2008) 299-316.

[13] H.C. Wu, The karush-kuhn-tucker optimality conditions in multiobjective programming problems with interval-valued objective functions. Eur. J. Oper. Res. 196 (2009) 49-60. 